\title{
Evaluating changes in radiation treatment volumes from post-operative to same-day planning MRI in High-grade gliomas
}

Colin E Champ ${ }^{1 *}$, Joshua Siglin ${ }^{1}$, Mark V Mishra ${ }^{1}$, Xinglei Shen ${ }^{1}$, Maria Werner-Wasik', David W Andrews², Sonal U Mayekar ${ }^{1}$, Haisong Liu ${ }^{1}$ and Wenyin Shi ${ }^{1}$

\begin{abstract}
Background: Adjuvant radiation therapy (RT) with temozolomide (TMZ) is standard of care for high grade gliomas (HGG) patients. RT is commonly started 3 to 5 weeks after surgery. The deformation of the tumor bed and brain from surgery to RT is poorly studied. This study examined the magnitude of volume change in the postoperative tumor bed and the potential impact of RT planning.

Method and materials: This study includes 24 patients with HGG who underwent craniotomy and adjuvant RT with TMZ at our institution. All patients had immediate postoperative MRI and repeat MRI during the day of RT simulation. Gross tumor volumes (GTV), clinical target volumes (CTV) of initial 46 Gy (CTV1) and boost to 60 Gy (CTV2) were contoured on both sets of MRls according to RTOG (Radiation Therapy Oncology Group) guidelines. For patients who recurred after RT, the recurrence pattern was evaluated.
\end{abstract}

Results: An average of 17 days elapsed between immediate and delayed MRIs. GTV1 (FLAIR abnormality and tumor bed) decreased significantly on the delayed MRI as compared to immediate post-operative MRI (mean $=30.96 \mathrm{cc}$, $p=0.0005$ ), while GTV2 (contrast-enhanced T1 abnormality and tumor bed) underwent a non-significant increase (mean $=6.82 \mathrm{cc}, \mathrm{p}=0.07$ ). Such changes lead to significant decrease of CTV1 (mean decrease is 113.9cc, $p<0.01$ ), and significant increase of CTV2 (mean increase is $32.5 \mathrm{cc}, \mathrm{p}=0.05$ ). At a median follow-up of 13 months, 16 patients (67\%) progressed, recurred, or died, with a progression-free survival time of 13.7 months. Twelve patients failed within all CTVs based on immediate and delayed MRIs, while one patient recurred outside of CTV2 based on immediate post-operative MRI, but within the CTV2 defined on delayed MRI.

Conclusion: The postoperative tumor bed of HGGs undergoes substantial volumetric changes after surgery. Treatment planning based on delayed MRI significantly reduces the volume of treated brain tissue without local control detriment. The marked reduction of volume treated to $46 \mathrm{~Gy}$ based on delayed MRI scan, could result in increased sparing of organs at risk. There may be a small risk of inadequate radiation field design if radiation planning is based on immediate post-operative MRI.

Keywords: High-grade glioma, Radiotherapy planning, Same-day MRI, Glioblastoma multiforme, Anaplastic astrocytoma

\footnotetext{
* Correspondence: Colin.Champ@jeffersonhospital.org

${ }^{1}$ Department of Radiation Oncology, Kimmel Cancer Center and Jefferson

Medical College of Thomas Jefferson University, 111 S. 11th Street,

Philadelphia, PA 19107, USA

Full list of author information is available at the end of the article
} 


\section{Background}

High-grade gliomas (HGG), including World Health Organization (WHO) grade III anaplastic astrocytoma (AA) and grade IV glioblastoma multiforme (GBM), occur in approximately 5 cases per 100,000 people in the US. HGG are the most frequently occurring gliomas, with GBM comprising $53.7 \%$ of all new cases and AA accounting for $6.7 \%$ [1].

Maximum safe resection is standard initial treatment for HGG, and the extent of resection is correlated with improved outcomes [2]. Several studies have shown that post-operative radiation therapy (RT) alone or combined with chemotherapy improves survival in HGG $[3,4]$. RT was historically delivered via a whole brain approach. However, Shapiro et al. showed equivalence in survival in patients treated with whole brain RT to 43 Gray (Gy) with a cone-down to $60 \mathrm{~Gy}$, resulting in a shift in treatment paradigm [5]. Further retrospective data from M.D. Anderson for HGG supported the efficacy of reduced field RT [6]. This approach is further supported by patterns of failure studies which, using combinations of biopsy, brain computed tomography $(\mathrm{CT})$, brain magnetic resonance imaging (MRI), and autopsy information, have uniformly indicated that the predominant failure pattern for HGG patients is within a 2-cm margin of the tumor volume [7-9].

Given the use of reduced field, conformal RT, it is becoming increasingly important to accurately delineate the post-operative tumor bed. Recent standards for RT for HGG as denoted in Radiation Therapy Oncology Group (RTOG) 0825 guidelines recommend use of a CT-based simulation merged to a pre or post-operative MRI [10]. Guidelines on radiation target volume delineation remain unclear with some authors advocating for smaller margins than those described in the protocol [11,12]. In addition, guidelines on optimal timing of planning MRI are limited as well. The recommended starting time for radiation is 3-5 weeks (21-35 days) after surgery [10]. The brain deformation and change in the tumor bed during this time period is poorly understood. We hypothesized that large changes in the tumor bed and T2/FLAIR abnormality would occur between the time of surgery and initiation of RT. Therefore, a delayed MRI at the time of RT planning may more accurately reflect the tumor bed at time of RT, and potentially allow us to spare more normal tissue with no decrease in local tumor control. The purpose of this study was to prospectively evaluate the changes in treatment volumes between immediate post-operative MRI and same-day MRI at time of RT planning in an attempt to spare more normal tissue.

\section{Methods}

\section{Study population}

At our institution, all patients with HGG who undergo surgical resection and biopsy have immediate postoperative
MRI (within 48 hours) to assess the extent of resection. We prospectively obtained a delayed MRI, which was performed at the time of RT planning, on 24 consecutive patients from July 19, 2010 to June 13, 2011, with pathologically confirmed HGG treated at our institution in an Institutional Review Board (IRB) approved protocol. All patients underwent craniotomy with resection or biopsy and were treated with temozolomide during RT and after. All data and medical records were reviewed comprehensively in accordance with the Health Insurance Portability and Accountability Act, our institutional IRB, and with the Helsinki Declaration of 1975, as revised in 2000.

\section{Contoured tumor volumes and treatment}

Patients were simulated in the supine position with an aquaplast head mask. CT scan without intravenous contrast was obtained on a GE LightSpeed CT simulator (GE Healthcare, Chalfont St. Giles, UK) and 2.5-mm slices were obtained. Immediate post-operative MRI was obtained within 48 hours after craniotomy. Same-day MRI was obtained on the day of treatment planning. All patients underwent thin-cut, 1-1.5 mm slice thickness MRI imaging, including pre- and post-contrast T1weighted and T2/FLAIR sequences. All images sets were imported into Elekta Focal Treatment Planning Software (Version 4.3.1, Stockholm, Sweden) and fused.

Target volume delineation was based on planning CT fusion with immediate and delayed MRI images. This provided us with fused images with high spatial fidelity (CT) and anatomic resolution (all MRI pulse sequences) upon which we could base target volume paired comparisons as well as design optimal treatment plans. The initial volume received a dose of $46 \mathrm{~Gy}$. The initial gross tumor volume (GTV1) was defined as tumor bed and T2/FLAIR abnormality. Clinical tumor volume 1 (CTV1) was GTV1 with a 2-cm expansion, modified to avoid barriers of spread (i.e. bone, falx cerebri, ventricles). Planning tumor volume 1 (PTV1) was a CTV1 with a 0.5-cm uniform expansion. GTV2, which is the boost volume to $60 \mathrm{~Gy}$, was defined as tumor bed and T1 post-contrast enhancement. CTV2 was a 2-cm uniform expansion of GTV2, modified to avoid barriers of spread. PTV2 was a 0.5 - $\mathrm{cm}$ uniform expansion of CTV2. All the contours were generated on both immediate and delayed MRIs. All contours were done by a single operator (C.C.) and confirmed by two expert observers (W.S. and J.S.) in order to limit interobserver variability. Both the operator and observers were blinded to whether the MRIs were post-operative or delayed and the MRIs were presented in a random manner to reduce recall bias.

\section{Power calculation}

After retrospectively reviewing and calculating mean volume changes in 5 patients treated at our institution, and 
based on an estimated standard deviation of $25 \%$, we required 24 patients to detect a difference of $30 \%$ in the CTV treatment volume with a two-sided hypothesis test at the 0.05 level of significance. These values were calculated at initial study design.

\section{Data analysis}

Current RT treatment guidelines for HGG in RTOG protocols and many US institutions depend largely on T2/FLAIR and T1 post-contrast enhancement which led us to analyze target changes over time defined by these two standard pulse sequences. Treatment margins are based solely on these MRI findings and we set out to elucidate any changes between scans occurring in the immediate post-operative period and those at the time of treatment planning, often occurring 2-4 weeks after craniotomy. Student's paired $t$-test was performed to compare the post-operative and delayed treatment volumes.

Recurrence patterns at interim analysis were then compared by fusion of the recurrence MRI with both treatment plans and all corresponding images. Recurrence was assessed individually on surveillance MRI. The site of recurrence was contoured on planning software and then compared with each blinded treatment MRI. Progression-free survival time was calculated using the Kaplan-Meier (KM) method. Location of recurrence within GTV1/2 and CTV1/2 based on post-operative MRI were compared to these volumes based on same-day MRI. Recurrence was based primarily on surveillance MRIs, which are reviewed at our weekly multidisciplinary brain tumor conference involving members from several institutional departments, including radiation oncology, neuro-oncology, neurosurgery, and neuroradiology. Biopsy-proven recurrence is not required in our institution if it is felt that imaging results show unequivocal progression by consensus of the board. Student's paired $t$-test was performed to compare recurrence patterns between postoperative and delayed treatment volumes.

Statistical analyses were performed using the Stata Statistical package (version IC 11.1, Texas). Logistic regression was performed to identify patient and tumor variables predicting for a $>30 \%$ change in CTV between the post-operative MRI and delayed MRI for both T1 and T2/FLAIR imaging sequences. Variables included in the analysis were steroid usage (dexamethasone), tumor histology, and extent of resection (gross total, subtotal, and biopsy). P-value of less than 0.05 was considered statistically significant.

\section{Results}

In total, 16 patients with GBM and 8 with AA were included in the analysis. The median age at the initiation of RT was 57 years old (range 22-78). Please refer to Table 1 for patient characteristics. Treatment-planning CT scans of the brain were obtained a median of 17 days after craniotomy (range 7-32) and merged with immediate and delayed MRI for planning (see Table 2). While our institution generally prefers treatment planning 2 weeks after craniotomy in an effort to initiate treatment during postoperative week 3 , this was not always possible due to extenuating patient circumstances.

\section{Volumetric analysis}

There were significant changes in both $\mathrm{T} 1$ postcontrast enhancement and T2/FLAIR abnormality from immediate post-operative MRI to delayed MRI scans in all patients. T2/FLAIR-based GTV1 decreased in 23 patients, with an average decrease of $22.1 \%$ (mean = $30.96 \mathrm{cc}$, range -140.15 to $-0.33, \mathrm{p}=0.0005)$. CTV1 had a $16.9 \%$ average decrease in volume $($ mean $=113.86 \mathrm{cc}$, range -347.70 to $36.73, \mathrm{p}=0.000003)$.

\section{Table 1 Patient demographics}

\begin{tabular}{|c|c|c|c|c|c|}
\hline Patient & Age & Histology & Location & Surgery & $\begin{array}{c}{ }^{*} \text { Dexamethasone } \\
\text { treatment }\end{array}$ \\
\hline 1 & 52 & $\mathrm{AA}$ & (R) Parietal & STR & No \\
\hline 2 & 56 & AA & (L) Parietal & GTR & Yes \\
\hline 3 & 58 & AA & (L) Temporal & STR & No \\
\hline 4 & 64 & AA & (L) Temporal & STR & No \\
\hline 5 & 70 & AA & (R) Temporal & STR & Yes \\
\hline 6 & 22 & $\mathrm{AA}$ & (R) Frontal & STR & Yes \\
\hline 7 & 36 & AA & (L) Frontal & GTR & Yes \\
\hline 8 & 59 & AA & (L) Frontal & GTR & No \\
\hline 9 & 56 & GBM & (L) Parietal & STR & No \\
\hline 10 & 78 & GBM & (R) Parietal & STR & No \\
\hline 11 & 61 & GBM & (L) Temporal & STR & Yes \\
\hline 12 & 59 & GBM & (L) Parietal & STR & Yes \\
\hline 13 & 70 & GBM & (R) Temporal & $B X$ & Yes \\
\hline 14 & 48 & GBM & (L) Frontal & STR & No \\
\hline 15 & 49 & GBM & (R) Frontal & STR & Yes \\
\hline 16 & 66 & GBM & (L) Temporal & STR & No \\
\hline 17 & 66 & GBM & (R) Temporal & STR & Yes \\
\hline 18 & 50 & GBM & (R) Frontal & GTR & No \\
\hline 19 & 46 & GBM & (L) Temporal & STR & Yes \\
\hline 20 & 87 & GBM & (R) Frontal & STR & No \\
\hline 21 & 35 & GBM & (R) Thalamus & STR & No \\
\hline 22 & 75 & GBM & (L) Frontal & STR & Yes \\
\hline 23 & 54 & GBM & (R) Frontal & STR & Yes \\
\hline 24 & 52 & GBM & (L) Frontal & STR & No \\
\hline
\end{tabular}

Abbreviations: AA Anaplastic astrocytoma, GBM Glioblastoma multiforme, STR Subtotal resection, GTR Gross total resection, BX Biopsy, $L$ Left, $R$ Right, *At time of MRI. 
Table 2 Treatment volumes

\begin{tabular}{|c|c|c|c|c|c|c|c|c|c|}
\hline Patient & MRI Span (Days) & GTV1 $\Delta$ & $\%$ & GTV2 $\Delta$ & $\%$ & CTV1 $\Delta$ & $\%$ & CTV2 $\Delta$ & $\%$ \\
\hline 1 & 8 & -40.7 & 42 & 6.9 & 37 & -139.4 & 26 & 37.9 & 16 \\
\hline 2 & 10 & -0.3 & 1 & 5.2 & 35 & -13 & 5 & 18.2 & 8 \\
\hline 3 & 7 & -16.4 & 35 & 3 & 26 & -77 & 20 & 16 & 8 \\
\hline 4 & 15 & -3.9 & 2 & 6.6 & 67 & -49.9 & 5 & 47.6 & 26 \\
\hline 5 & 19 & -19.7 & 14 & 13.9 & 25 & -49.4 & 7 & 33.9 & 7 \\
\hline 6 & 26 & -25.9 & 37 & -17 & 37 & -125.1 & 26 & -89.8 & 24 \\
\hline 7 & 17 & -10.1 & 7 & -5 & 4 & -93.1 & 12 & -35.8 & 6 \\
\hline 8 & 13 & -2.4 & 36 & -0.3 & 14 & -27.5 & 17 & -3.4 & 3 \\
\hline 9 & 13 & -12.4 & 27 & 3.3 & 44 & -83.1 & 20 & 15.1 & 22 \\
\hline 10 & 27 & -21.8 & 33 & 1.9 & 31 & -154.6 & 23 & 18 & 12 \\
\hline 11 & 17 & -52.7 & 34 & 22 & 39 & -195.9 & 24 & 198.8 & 83 \\
\hline 12 & 11 & -12.4 & 18 & 2.2 & 7 & -69.4 & 14 & 1.4 & 0 \\
\hline 13 & 15 & -14.3 & 16 & 22.6 & 37 & -16.9 & 3 & 136.9 & 31 \\
\hline 14 & 16 & 36.9 & 60 & 16.2 & 107 & 184.9 & 24 & 85.8 & 37 \\
\hline 15 & 12 & -35.8 & 31 & -10.8 & 27 & -95.8 & 18 & -43 & 12 \\
\hline 16 & 32 & -72 & 63 & -0.2 & 1 & -282.8 & 42 & 45.2 & 16 \\
\hline 17 & 14 & -130.9 & 54 & 14.6 & 20 & -347.7 & 34 & 64.4 & 12 \\
\hline 18 & 28 & -35.5 & 44 & 1.8 & 6 & -160.3 & 31 & -14.6 & 5 \\
\hline 19 & 16 & 11.6 & 100 & -13.4 & 54 & 36.7 & 13 & -5.1 & 3 \\
\hline 20 & 19 & -140.1 & 56 & 6.3 & 19 & -300.6 & 28 & 31 & 9 \\
\hline 21 & 31 & -17 & 13 & -10.6 & 9 & -101.1 & 14 & -82.2 & 12 \\
\hline 22 & 22 & -2 & 3 & -2 & 21 & -10.1 & 2 & -10.5 & 6 \\
\hline 23 & 14 & -5.2 & 3 & 73.3 & 121 & -27 & 3 & 249.4 & 51 \\
\hline 24 & 16 & -46.3 & 26 & 19.4 & 36 & -164.6 & 19 & 65.4 & 16 \\
\hline Average & 17 & -31 & 22 & 6.8 & 22 & -113.9 & 17 & 32.5 & 12 \\
\hline
\end{tabular}

Abbreviations: Post-op Post-operative, MRI Magnetic resonance imaging, GTV1 Gross tumor volume treated to 46 Gy, CTV1 Clinical tumor volume treated to 46 Gy, GTV2 Gross tumor volume treated to $60 \mathrm{~Gy}$, CTV2 Clinical tumor volume treated to $60 \mathrm{~Gy}$, (-) values indicate volume was smaller on same-day MRI, (+) values indicate volume was larger on delayed MRI.

T1 post-contrast enhancement-based GTV2 underwent an average increase of $22 \%$ (mean $=6.82 \mathrm{cc}$, range -13.44 to $73.26, \mathrm{p}=0.07)$. This resulted in a $12 \%$ increase of CTV2 (mean $=32.52 \mathrm{cc}$, range -89.83 to $249.45, \mathrm{p}=0.05)$. Thirteen patients in total had a $30 \%$ or greater increase in GTV1 ( $\mathrm{n}=9 / 16 \mathrm{GBM}$ patients), while 11 patients had a $20 \%$ or greater increase in CTV1 $(\mathrm{n}=8 / 16$ GBM patients, $\mathrm{n}=3 / 8$ AA patients). Several lesions had a striking change in size, including a CTV2 increase of $83 \%$ (Figure $1 \mathrm{C}$ and D), a CTV2 increase of $51 \%$ (Figure 2), and a CTV1 decrease of $42 \%$; all were GBMs.

In subgroup analysis, AA GTV1 volume underwent a $16 \%$ decrease $($ mean $=14.94 \mathrm{cc}$, range 0.33 to 40.73 , $\mathrm{p}=0.02)$. CTV1 decreased by $13 \%$ (mean $=71.81 \mathrm{cc}$, range -12.96 to $-139.43, \mathrm{p}=0.002$ ). However, changes in GTV2 $(5 \%$, mean $=1.67 \mathrm{cc}$, range 0.30 to 16.96 , $\mathrm{p}=0.63)$ and CTV2 $(1 \%$, mean $=3.05 \mathrm{cc}$, range -89.83 to $47.60, \mathrm{p}=0.86)$ were non-significant.
In contrast, GTV1 for GBM patients underwent broad fluctuations in the post-operative and delayed GTV volumes, with a decrease in volume of $32.82 \%$ (mean = $38.97 \mathrm{cc}$, range 23.19 to $189.32, \mathrm{p}=0.003$ ). Similarly in significance, CTV1 decreased by an average of $20.01 \%$ $($ mean $=134.88 \mathrm{cc}$, range -300.64 to $36.73, \mathrm{p}=0.0008)$. GTV2 underwent lesser changes that trended toward significance, with an increase of $23.44 \%$ (mean $=9.40 \mathrm{cc}$, range -13.44 to $73.26, \mathrm{p}=0.09$ ). CTV2 reached statistical significance with an increase in size by $14.43 \%$ ( mean $=47.25 \mathrm{cc}$, range -82.18 to $249.50, \mathrm{p}=0.05$ ).

When comparing volume changes with steroid usage, tumor histology, and type of resection, no significant effects in treatment volume were appreciated when logistic regression was performed.

\section{Recurrence patterns}

At a median follow-up of 13 months, 16 patients progressed or died. One patient recurred distantly and 2 


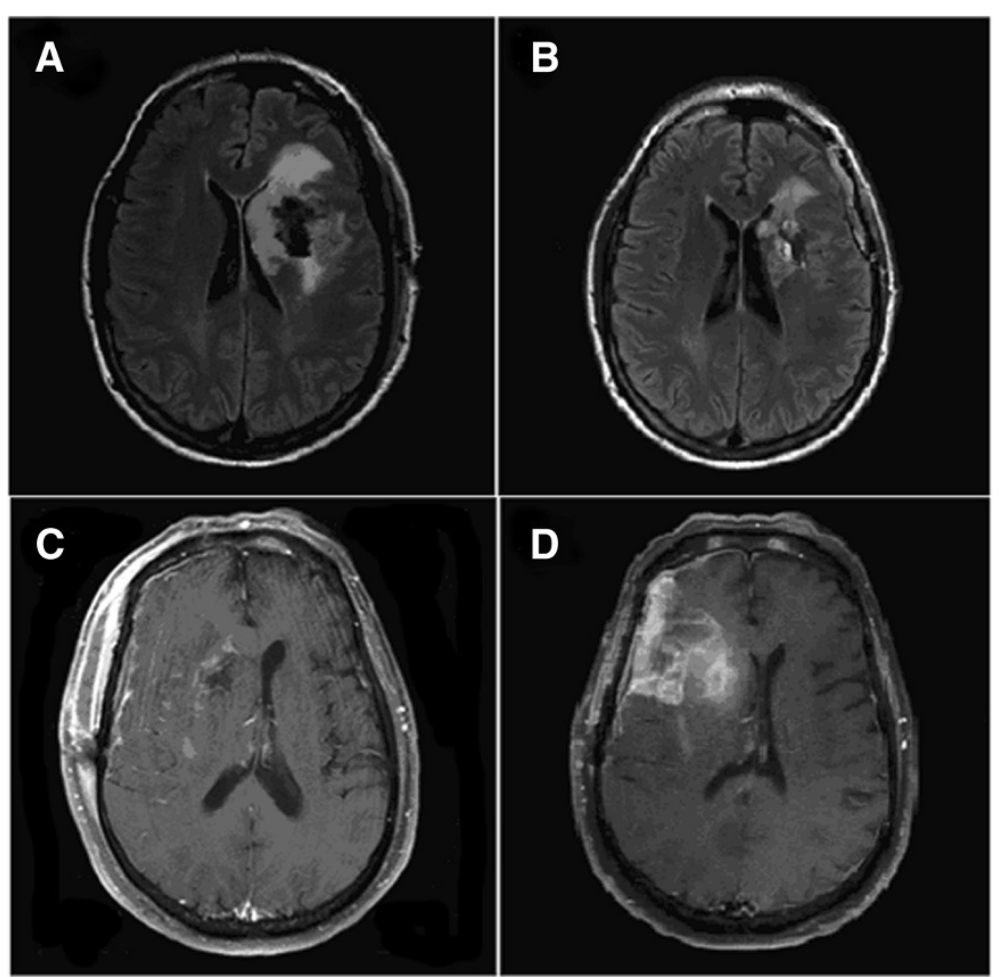

Figure 1 Changes in volume on T2/FLAIR and T1 contrast-enhanced MRI: A. T2/FLAIR MRI performed 1 day after craniotomy. B. T2/ FLAIR MRI performed 2 weeks after craniotomy. Another patient with C. T1 contrast-enhanced MRI performed 1 day after craniotomy. D. T1 contrast-enhanced MRI performed 2 weeks after craniotomy.

died with no sign of progression. Progression-free survival time was 13.7 months, on KM analysis (Figure 3). Failure was within CTV1 and CTV2 based on both immediate and delayed imaging in $86 \%$ of patients $(n=12 / 14)$. One patient failed outside of CTV2 based on immediate post-operative MRI only and no patients failed outside of CTV1 or CTV2 based on same-day imaging. This was not statistically significant $(\mathrm{p}=0.34)$. Though not significant, $46 \%$ of patients $(n=6)$ failed outside of one or more GTV volumes on postoperative MRI and $39 \%$ of patients $(\mathrm{n}=5)$ failed outside of one or more GTV on same-day MRI ( $\mathrm{p}=0.35)$.

\section{Discussion}

Long term survival of patients with HGG remains poor due to local recurrence after treatment, with the vast majority occurring within a $2-\mathrm{cm}$ area of the presurgical tumor margin, emphasizing the importance of accurately defined treatment volumes [7-9]. Guidelines for treatment planning have been evolving in recent RTOG protocols, which are used as guidelines for treatment planning by many institutions within the US and internationally. While the recent RTOG 0825 protocol required CT-based planning for RT with post-operative MRI fusion to ensure accurate target delineation, only three years earlier RTOG 0525 used pretreatment MRIs for treatment planning [10]. While CT often displays the surgical tumor bed, MRI presents the planning physician with further tools to elucidate tumor bed and surrounding edema [13]. Contrast-enhanced T1 MRI best evaluates residual tumor and areas of blood-brain barrier breakdown, while T2/FLAIR enhancement best evaluates tissue edema $[14,15]$.

Our data shows that the CTV1, including the resection cavity and surrounding edema, changes extensively in both GBM and AA patients in the small time interval between surgery and RT planning, with same-day planning MRI elucidating a more accurate representation of the lesion and decrease in surgical edema. CTV2 also changed significantly for combined AA and GBM, but only for GBM patients on subset analysis. Twenty-three of 24 CTV1 volumes decreased in size within an average of 17 days between post-operative MRI and treatment planning MRI. This decrease in size can potentially spare proximal organs at risk and spares normal brain tissue from being treated to the initial dose of $46 \mathrm{~Gy}$. In contrast, CTV2 increased in size in 16 of 24 patients, which could potentially result in improved tumor bed coverage during the treatment boost in comparison to immediate post-operative MRI. Nearly a third of all patients had over a $25 \%$ change in CTV2 with planning MRI performed in 17 days or less after craniotomy, including 

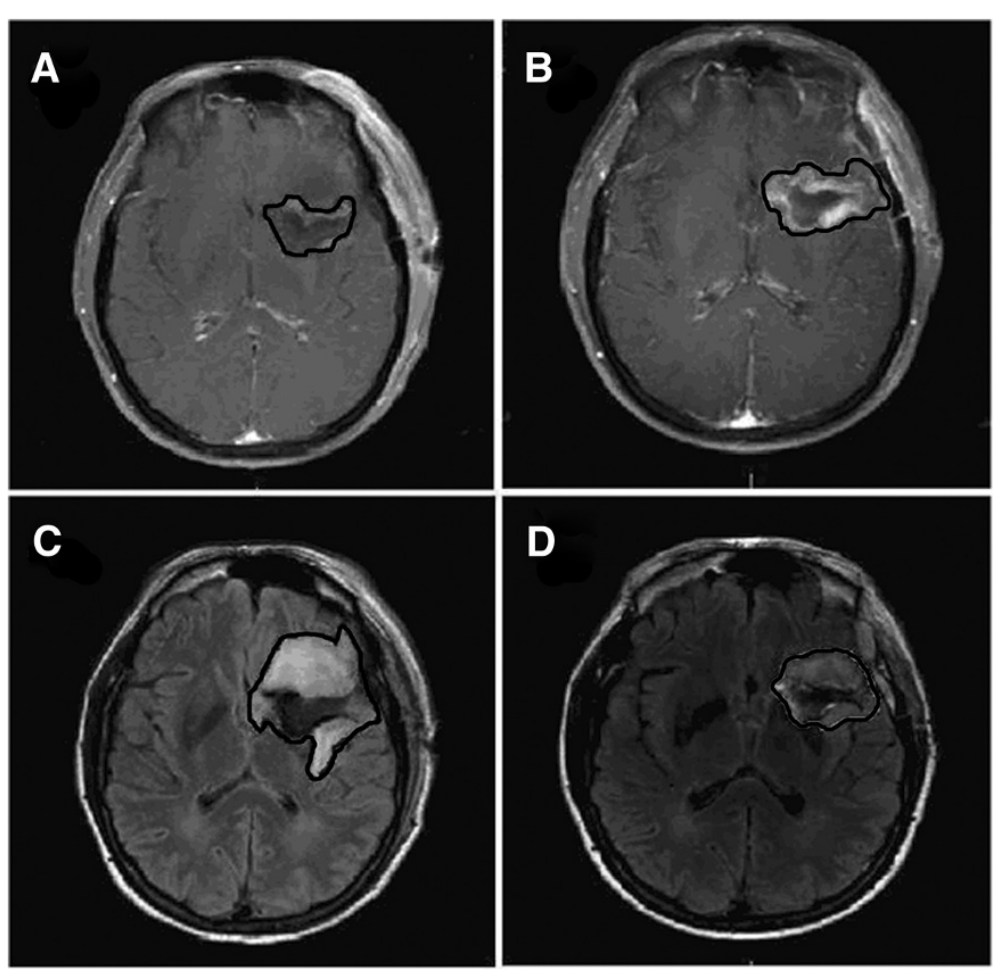

Figure 2 Changes of GTV on T2/FLAIR and contrast-enhanced MRI: A. GTV2 on post-operative T1 contrast-enhanced MRI. B. GTV2 on delayed T1 contrast-enhanced MRI, C. GTV1 on post-operative T2/FLAIR MRI. D. GTV1 on delayed T2/FLAIR MRI.

2 patients with a greater than $50 \%$ change in their volumes.

Our data also shows that treating with RT based on same-day MRI does not result in increased rates of tumor recurrence outside of the treatment volume, as no failures were seen outside of the CTV volumes based on same-day MRI. In fact, only one patient had disease recurrence locally which was outside of CTV based on post-operative imaging only. Therefore, while less

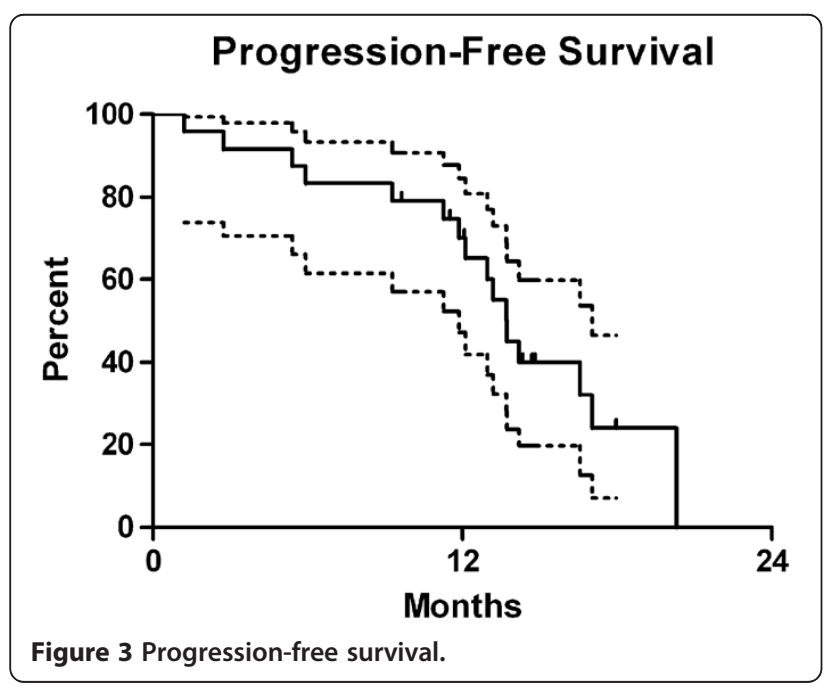

volume of tissue is irradiated when planning is based on same-day MRI versus immediate post-operative MRI, it is unlikely that treatment based on same-day MRI results in an increase in tumor-miss. These changes could result in alterations in treatment volumes, resulting in a large impact for future RTOG and other protocols, which may be based on imaging sets that do not accurately define the tumor bed volume.

Similar findings regarding volume changes were presented at the 2010 American Society for Radiation Oncology Annual Meeting, revealing large variations in surgical cavities with both expansion and shrinkage occurring mostly within the first two weeks after craniotomy for HGG patients. This group of investigators also demonstrated a benefit in local control and a decrease in radiation injury in patients with planning MRI delayed for over two weeks [16]. While we cannot determine a benefit in local control as the aggressive nature of these tumors results in frequent local recurrence, it appears that when MRI is delayed over two weeks, normal tissue can be spared with no detriment on local control.

While RTOG 0825 has a clear set of guidelines concerning treatment margins, some practitioners advocate tighter margins [11,12]. However, when reviewing our data, caution should be employed with this technique as it introduces the potential of missing gross tumor, as our data indicates that GTV increases even in the short time 
period between post-operative and same-day MRI, timing of MRI may become more important for practitioners of limited-margin treatment to limit the possibility of tumor miss.

While we have shown that the resection cavity undergoes extensive volumetric changes in the short interval between surgical resection and RT planning, several studies have illustrated volumetric changes throughout treatment. Shukla et al. have shown that at week 5 of treatment, $12 / 15$ patients with unifocal disease had a median reduction of $54.85 \mathrm{cc}$ in tumor volumes on T2weighted MRI [17]. As a result, they suggest midtreatment re-planning MRI, potentially to define boost fields. Tsien et al. prospectively followed 21 patients who were rescanned at week 3 during RT [18]. They found an increase in GTV in 3/12 patients with GBM, with all cases requiring increase in size of treatment fields. A multi-institutional trial examining volume changes midway through treatment of GBMs showed a change in GTV in $80 \%$ of cases [19]. Given the increasing impetus to reduce treatment volumes recently, further research in this area may be warranted.

It is possible that the treatment volume undergoes several changes as the resection space contracts, surrounding edema waxes and wanes, and the actual tumor volume changes, and no minimal amount of reasonable reimaging could monitor this change. While the decrease in treatment volume seen on T2/FLAIR imaging in our study was likely from a reduction in peri-tumor edema, the increase in GTV2 was somewhat unexpected. This change may represent evolution of blood products, principally methemoglobin, post-operative changes which have been documented to occur between days 5 and 14 post-operatively, and/or tumor progression [20]. Optimal imaging times remain unknown but target volumes may be least ambiguous after 14 days postoperatively. As a result, we recommend that the MRI used to define the planning volume should be delayed to the time of CT simulation, with the treatment to start as soon as possible to reduce the potential for further changes that might negatively impact the effectiveness of treatment.

\section{Conclusions}

The results of this study illustrate the changes that the post-operative tumor bed of high-grade gliomas undergoes prior to RT and the important effect on target delineation during treatment planning. The marked reduction of volume treated to 46 Gy based on sameday versus post-operative MRI may result in better sparing of normal brain tissue without a detriment in local control. Additionally, the increased CTV2 volume may result in better tumor coverage, avoiding potential geographic miss in treatment based on immediate post- operative MRI scan. Given the differences demonstrated in treatment volume at varying MRI time points, further attempts should be made to correlate treatment planning with patterns of failure to maximize local control while minimizing toxicity.

\section{Competing interests}

The authors declare that they have no competing interests.

\section{Authors' contributions}

CC and WS designed the study, performed radiation planning, analyzed data, and drafted the manuscript. JS, MM, SM helped perform radiation planning, analyzed data, and helped draft the manuscript. MW, DA, XS, HL helped analyze data and draft the manuscript. All authors read and approved the final manuscript.

\section{Funding support}

Research was supported in part by the Kimmel Cancer Center's NCl Cancer Center Support Grant P30 CA56036.

\section{Author details}

${ }^{1}$ Department of Radiation Oncology, Kimmel Cancer Center and Jefferson Medical College of Thomas Jefferson University, 111 S. 11th Street,

Philadelphia, PA 19107, USA. ${ }^{2}$ Neurological Surgery, Kimmel Cancer Center and Jefferson Medical College of Thomas Jefferson University, Philadelphia, PA, USA.

Received: 15 June 2012 Accepted: 18 December 2012

Published: 21 December 2012

\section{References}

1. 2011 CBTRUS Statistical Report: Primary Brain and Central Nervous System Tumors Diagnosed in the United States in 2004-2007. Central Brain Tumor Registry of the United States. 2011, (Accessed June 7, 2011, at http://www.cbtrus.org/2011-NPCR-SEER/WEB-0407-Report-3-3-2011.pdf.

2. Sanai N, Berger MS: Glioma extent of resection and its impact on patient outcome. Neurosurgery 2008, 62:753-764. discussion 264-756.

3. Wen PY, Kesari S: Malignant gliomas in adults. N Engl J Med 2008 359:492-507.

4. Stupp R, Mason WP, van den Bent MJ, Weller M, Fisher B, Taphoorn MJ, Belanger K, Brandes AA, Marosi C, Bogdahn U, et al: Radiotherapy plus concomitant and adjuvant temozolomide for glioblastoma. N Engl J Med 2005, 352:987-996.

5. Shapiro WR, Green SB, Burger PC, Mahaley MS Jr, Selker RG, VanGilder JC, Robertson JT, Ransohoff J, Mealey J Jr, Strike TA, et al: Randomized trial of three chemotherapy regimens and two radiotherapy regimens and two radiotherapy regimens in postoperative treatment of malignant glioma. Brain Tumor Cooperative Group Trial 8001. J Neurosurg 1989, 71:1-9.

6. Garden AS, Maor MH, Yung WK, Bruner JM, Woo SY, Moser RP, Lee YY: Outcome and patterns of failure following limited-volume irradiation for malignant astrocytomas. Radiother Oncol 1991, 20:99-110.

7. Wallner KE, Galicich JH, Krol G, Arbit E, Malkin MG: Patterns of failure following treatment for glioblastoma multiforme and anaplastic astrocytoma. Int J Radiat Oncol Biol Phys 1989, 16:1405-1409.

8. Oppitz U, Maessen D, Zunterer H, Richter S, Flentje M: 3D-recurrencepatterns of glioblastomas after CT-planned postoperative irradiation. Radiother Oncol 1999, 53:53-57.

9. Chan JL, Lee SW, Fraass BA, Normolle DP, Greenberg HS, Junck LR, Gebarski SS, Sandler HM: Survival and failure patterns of high-grade gliomas after three-dimensional conformal radiotherapy. J Clin Oncol 2002, 20:1635-1642.

10. RTOG 0825: Phase III Double-Blind Placebo-Controlled Trial of Conventional Concurrent Chemoradiation and Adjuvant Temozolomide Plus Bevacizumab versus Conventional Concurrent Chemoradiation and Adjuvant Temozolomide in Patients with Newly Diagnosed Glioblastoma. 2011, Accessed April 5, 2011, at www.rtog.org.

11. McDonald MW, Shu H-KG, Curran WJ, Crocker IR: Pattern of Failure After Limited Margin Radiotherapy and Temozolomide for Glioblastoma. International journal of radiation oncology, biology, physics 2011, 79:130-136. 
12. Chang EL, Akyurek S, Avalos T, Rebueno N, Spicer C, Garcia J, Famiglietti R, Allen PK, Chao KSC, Mahajan A, et al: Evaluation of Peritumoral Edema in the Delineation of Radiotherapy Clinical Target Volumes for Glioblastoma. International Journal of Radiation Oncology*Biology*Physics 2007, 68:144-150.

13. Dillon WP: Imaging of central nervous system tumors. Curr Opin Radiol 1991, 3:46-50.

14. Castillo M: Contrast enhancement in primary tumors of the brain and spinal cord. Neuroimaging Clin N Am 1994, 4:63-80.

15. Bronen RA, Sze G: Magnetic resonance imaging contrast agents: theory and application to the central nervous system. J Neurosurg 1990, 73:820-839.

16. Iuchi T, Hatano K, Kodama T, Tohyama N, Kojima T, Sakaida T, Kawasaki K, Hasegawa Y: Brain Deformation after Planning for IMRT may Affect the Outcome of Patients with Glioblastoma. International journal of radiation oncology, biology, physics 2010, 78:S278-S279.

17. Shukla D, Huilgol NG, Trivedi N, Mekala C: T2 weighted MRI in assessment of volume changes during radiotherapy of high grade gliomas. J Cancer Res Ther 2005, 1:235-238.

18. Tsien C, Gomez-Hassan D, Ten Haken RK, Tatro D, Junck L, Chenevert TL, Lawrence T: Evaluating changes in tumor volume using magnetic resonance imaging during the course of radiotherapy treatment of high-grade gliomas: Implications for conformal dose-escalation studies. Int J Radiat Oncol Biol Phys 2005, 62:328-332.

19. Manon R, Hui S, Chinnaiyan P, Suh J, Chang E, Timmerman R, Phan S, Das R, Mehta M: The impact of mid-treatment MRI on defining boost volumes in the radiation treatment of glioblastoma multiforme. Technol Cancer Res Treat 2004, 3:303-307.

20. Forsyth PA, Petrov E, Mahallati H, Cairncross JG, Brasher P, MacRae ME, Hagen NA, Barnes P, Sevick RJ: Prospective study of postoperative magnetic resonance imaging in patients with malignant gliomas. J Clin Oncol 1997, 15:2076-2081.

doi:10.1186/1748-717X-7-220

Cite this article as: Champ et al:: Evaluating changes in radiation

treatment volumes from post-operative to same-day planning MRI in High-grade gliomas. Radiation Oncology 2012 7:220.

\section{Submit your next manuscript to BioMed Central and take full advantage of:}

- Convenient online submission

- Thorough peer review

- No space constraints or color figure charges

- Immediate publication on acceptance

- Inclusion in PubMed, CAS, Scopus and Google Scholar

- Research which is freely available for redistribution 